\title{
A Randomised Trial of Conventional Care versus Radioablation (Stereotactic Body Radiotherapy) for Extracranial Oligometastases
}

\author{
V.Khoo*M.Hawkins ${ }^{\dagger M}$.Ahmed $¥ A . K$ Kirby $¥ N$.van

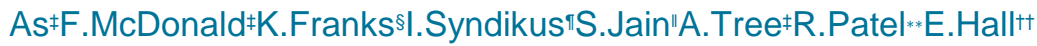

Background: Studies indicate SBRT treatment of oligometastatic disease is associated with higher rates of local control with acceptably toxicity [1,2]. A lack of randomised data in this area limits interpretation, preventing evaluation of the true benefit of adding SBRT to systemic therapy in this context. CORE investigates whether adding SBRT to standard therapy improves patient outcomes in common primary tumour sites where oligometastatic disease is encountered (NSCLC, breast and prostate). CORE aims to demonstrate: (1) feasibility of recruitment, (2) deliverability in a multi-centre setting and (3) activity of SBRT based on progression free survival (PFS) across three tumour types.

Methods: Eligible patients will have either primary NSCLC, breast or prostate cancerpresenting with $\leq 3$ extra-cranial, metachronous, oligometastases that are suitable for SBRT.

Randomised 1:1 to standard of care (SOC) or SOC + SBRT

Choice of SOC at local clinician's discretion, defined prior to randomisation (except local ablative therapies, i.e. surgery, RFA, cryotherapy.

Follow-up aligned with routine care up to 5 years.

Primary end point of PFS.

Results: CORE opened in October 2016 with 21 centres currently recruiting (18 UK, 3 Australia). Recruitment is currently on target with 88 patients randomised (66 prostate, 14 breast, 8 lung). Activities are now focussing on achieving equal recruitment across the three cohorts.

Future perspectives: The Trial Management Group are keen to build on the prostate experience and increase recruitment to the breast and lung cohorts. If the phase II aims of CORE are achieved, additional funding will be sought to roll the study into parallel tumour site-specific phase III trials.

\section{References}


[1] Corbin KS, Hellman S, Weichselbaum RR. Extracranial oligometastases: a subset of metastases curable with stereotactic radiotherapy. J Clin Oncol 2013;31(11):1384-90.

[2] Tree AC, Khoo VS, Eeles RA, Ahmed M, Dearnaley DP, Hawkins MA et al. Stereotactic body radiotherapy for oligometastases. Lancet Oncol 2013;14(1):e2837. 